\title{
Resurrecting melancholia
}

Fink M, Taylor MA. Resurrecting melancholia.

Objective: To define melancholia as a distinct mood disorder, identified by unremitting depressed mood, vegetative dysfunction, and psychomotor disturbances, verifiable by neuroendocrine tests, and treatable by electroconvulsive therapy and tricyclic antidepressants. Method: A review of the literature of two centuries finds descriptions of severe mood disorders, either depression or mania or circular, defined as 'melancholia.' In the 1980 diagnostic revision (DSM-III), melancholia was relegated to a features specifier only.

Results: DSM classification criteria develop heterogeneous patient samples that are neither guides to prognosis nor to treatment response, and confound studies of pathophysiology. Within the large population of mood disorders, a syndrome of melancholia is identifiable by specific behaviors, vegetative signs, and validated by neuroendocrine abnormalities (cortisolemia). Populations so identified are clinically homogeneous and have improved treatment responses. Patients meeting criteria for melancholia are now identified as psychotic depressed, geriatric depressed, postpartum psychosis, and pharmacotherapy resistant.

Conclusion: The review supports the establishment of melancholia by empirically derived criteria rather than by a checklist is an alternative to the major depression choice and offers an improved model for psychiatric classification.

\section{Fink ${ }^{1}$, M. A. Taylor ${ }^{2}$}

${ }^{1}$ Department of Psychiatry and Behavioral Science, SUNY at Stony Brook School of Medicine, St James, NY and ${ }^{2}$ Department of Psychiatry at the University of Michigan, Ann Arbor, MI, USA

Professors Fink and Taylor declare no conflict of interests

Key words: melancholia; classification; pathophysiology; hypercortisolemia; tricyclic antidepressant; electroconvulsive therapy

Max Fink, Department of Psychiatry and Behavioral Science, SUNY at Stony Brook School of Medicine, PO Box 457, St James, NY 11780, USA.

E-mail: mafink@attglobal.net

Presented at the Conference: 'Melancholia: Beyond DSM, Beyond Neurotransmitters', May 2-4, 2006, Copenhagen, Denmark.

\section{Introduction}

The DSM-III classification of psychiatric disorders of 1980 was a hoped-for improvement in nosology, as it sought an operationally defined system based on overt behaviors. In this and subsequent iterations, the core mood disorder became a monolithic 'major depressive disorder' with modifiers for differences in severity (e.g. psychotic or not), and for circumstances presumed to have clinical significance (e.g. abnormal bereavement, postpartum depression). The traditional concept of 'manicdepression' was separated into unipolar and bipolar categories. The well-established concept of melancholia was discarded, and laboratory tests were excluded from the criteria (1).

The diagnostic criteria that were formulated, however, are imprecise, inadequate for treatment decisions, and do not assure homogeneous populations in clinical trials, thereby missing opportunities to define the specific actions that are sought in new treatments. A cogent example is the evaluation of antidepressants in the STAR*D study (2). In a US government-supported multi-site collaborative effort, out-patients meeting criteria for non-psy- chotic unipolar depression were first treated with citalopram, and 30\% responded. The remaining patients were offered one of three antidepressant agents, to which an additional one-quarter responded, leaving half the subjects without symptomatic relief in repeated trials. The study does not offer effective guidelines for subject selection for these medications.

The out-patient population sample was heterogeneous. Although the severity of the illness was rated as moderate (HDRS (Hamilton Depression Rating Scale) $)_{17}$ score $\left.19 \pm 7.3\right), 17 \%$ reported suicide attempts, $76 \%$ suffered recurrent illnesses, the duration of the present episode averaged 30 months, the lifelong duration of illness was 17 years, and the subjects reported an average of seven episodes of illness. Features of anxious depression were recorded in $44 \%$ and atypical depression in $20 \%$. The criteria for 'major depression' failed to distinguish those with characterological and social influences on their illness from those with a distinctive biological basis. The heterogeneity of the sample is one explanation for the weak results.

Guidelines to establish diagnostic validity for a clinical condition, and therefore maximize homo- 
geneity, were offered by Robins and Guze (3). They defined a clinical syndrome as the delineation of characteristic signs and symptoms, supported by laboratory tests or biologic markers, with a characteristic clinical course and response to treatments. We applied these principles in an earlier assessment of catatonia. We described the characteristic motor signs in examination and rating scales, validated the syndrome by a lorazepam response test, and successfully treated patients with sedative anticonvulsant medications and convulsive therapy $(4,5)$.

Using a similar method, we propose the replacement of the present potpourri of mood disorders in DSM-III and DSM-IV that serves our patients poorly. We demarcate a prototypic syndrome of melancholia by specific psychopathology, laboratory tests, and effective treatments (1). The remaining mood disorders are categorized in a non-homogeneous class of non-melancholic disorders, awaiting the definition of other distinctive syndromes using the same principles. An example is offered by the analysis of atypical depression by Stewart et al. (6) in this issue (see pp. 58-71).

\section{The concept of melancholia}

A syndrome of 'madness' with 'bodily causes' has been clinically recognized for centuries. Except for two periods in Western history - the Middle Ages in Europe when church teachings dominated Western thought and in the 20th century when psychoanalytic notions usurped psychiatric thinking - melancholia was identified as a distinct periodic mood disorder with both manic and depressive phases and without progressive deterioration (1, 7-12). In 20th century classifications, psychoanalysts defined psychiatric illnesses as being of 'mental,' not brain origin, with depressive and manic periods as 'reactions' to personal experience. The classifications in DSM-I and DSM-II were based on this philosophy.

By the late 1960s, however, it was no longer considered acceptable to treat depressed patients as if they were all suffering from a single condition, as treatments were found to be effective for some patients and not for others (13-16). An assessment of 33 studies of medication treatments for depression, for example, could not find a diagnostic formulation that had predictive strength (17). The poor reliability of the existing classifications was clearly demonstrated in the strikingly different diagnostic conclusions reached by American and British psychiatrists examining the same patients $(18,19)$.
Melancholia has been ignored in each subsequent DSM revision. To acknowledge the many conditions that could be described, DSM-III listed 265 disorders, increased to 292 in DSM-IIIR, and to 295 in DSM-IV (20). A similar increase in the number of putative disorders is found in the ICD classification system (21).

\section{From major depression to melancholia}

In our present system of diagnosis, we identify patients who meet criteria for major depression by cross-sectional, unweighted features. Persons who feel saddened by life events or who are disgruntled and unhappy by virtue of life's vicissitudes or by their personality traits meet criteria for major depression if these moods are associated with decreased energy or interest. Their inclusion within the class of major depression confounds the results of clinical treatment trials and laboratory searches for an understanding of mood disorders. The large numbers of placebo responders in such samples attest to the need for a higher threshold to identify depressed persons that warrant somatic treatment $(1,2)$.

Quality of mood (unremitting apprehension and sadness), psychomotor disturbance (agitation or bradykinesia), multiple vegetative signs, and psychosis are essential elements in defining melancholia. The present psychiatric classification does not require these features in the diagnosis of a depressive illness.

Abnormal neuroendocrine tests and disturbed sleep EEG measures are common in melancholia. Hypercortisolemia is prominent, particularly when the melancholic patient is agitated or psychotic. When measured by the dexamethasone suppression test (DST), cortisol functions are abnormal when patients are ill, normalize with remission, and became abnormal again in relapse $(1,22,23)$. Similar findings, although less robust, are seen in thyroid function tests and sleep EEG measures (1). Although developed heuristically and without a central theory, these tests buttress the melancholia diagnosis. They are equally useful (e.g. similar in sensitivity and specificity) to the EEG, brain imaging, and serum prolactin levels in defining a seizure disorder.

In the psychopathological literature, melancholia is consistently described as a severe illness of acute onset with unremitting moods of apprehension and gloom, psychomotor disturbance, and vegetative signs. Psychosis, intermittent mania, and suicide intent are prominent features. A compelling picture of melancholia is offered by Falret in describing a circular insanity: 


\section{Fink and Taylor}

At the commencement of this phase... the patients begin to withdraw and now speak only rarely. Sometimes they express remorse over their previous condition... remaining all alone and motionless... they are now meek, and their humility may go so far as for them to refuse treatment in the belief that they do not deserve it. This despondency becomes more pronounced daily... [and] the patient is transformed into a statue... were he not coaxed to eat, the patient would not bother to seek food....

The thought processes and his movements are very slow; rarely this may result in complete cessation of all intellectual activity.... The face is pale; the features sag, suggesting dejection rather than anxiety.... Appetite is decreased, and the patient eats little; digestion is equally slow and defecation is laborious.

Nevertheless, there are a certain number of patients who present with specific preoccupations, among which we have noticed ideas of humility, of ruin, of being poisoned, or of guilt. (24)

We propose to reinstitute the definition of melancholia, set a duration criterion of two weeks, and add as secondary criteria the associated laboratory findings of dexamethasone non-suppression of cortisol, high nighttime cortisol levels, or decreased REM latency or other characteristic sleep abnormalities (Table 1).

Applying such criteria to the entities described in DSM-IV finds that patients with diverse syndromes as presently defined meet the criteria for melancholia (Table 2). Patients with mood disorders who do not meet criteria for melancholia comprise a large heterogeneous population best labeled for the present as within the non-melancholic mood disorders.

The proposed criteria for melancholia have face validity for high specificity. They make falsepositive diagnoses unlikely, and maximize the identification of homogeneous population samples for clinical trials and studies of pathophysiology. They meet the three principal criteria for a clinical syndrome delineated by Robins and Guze (3).

Table 1. Proposed diagnostic criteria for melancholia (1) (all must be present)

A. An episode of illness with reduced functioning characterized by an unremitting mood of apprehension and gloom that compromises normal daily activities and persists for at least 2 weeks

B. Psychomotor disturbance as agitation, retardation (including stupor and catatonia), or both

C. Vegetative signs (at least two).

D. At least one of the following:

Abnormal dexamethasone suppression or dexamethasone-suppressed corticotropin-releasing hormone test (DEX/CRH); high nighttime cortisol levels Decreased REM latency or other sleep abnormalities
Table 2. Proposed inclusions in melancholia

\begin{tabular}{lc}
\hline Melancholia & Non-melancholic mood disorder \\
\hline Psychotic depression & Characterological depression \\
Manic-depression & Reactive depressive disorders \\
Puerperal depression & Premenstrual dysphoria \\
Abnormal bereavement & \\
Depression with stupor or catatonia & \\
\hline
\end{tabular}

\section{Verification by psychopathology}

Almost all empirical studies over four decades report 'major depression' as encompassing different patient groupings with between $20 \%$ and $70 \%$ of the samples described as psychotic, melancholic, or endogenously depressed. The less homogeneous groups are younger, less severely ill, exhibit abnormal trait behavior, and meet criteria for anxiety disorder or atypical depression (1).

The strongest 'negative' study is that of Kendell $(25,26)$ who used data from hospital discharge forms collected by trainee psychiatrists at the Maudsley Hospital between 1949 and 1963. Kendell failed to find a bimodal distribution of patient characteristics and concluded that depressive disorders were best viewed as a continuum. Although the Maudsley report form did not include items needed to delineate melancholia and the inter-rater reliability of the raters is unknown, we looked carefully at the two factors that were delineated (Table 3). Kendell clearly identified a 'psychotic depression' group that closely matches criteria for the melancholia syndrome.

Over 70 other studies in the past quarter century refute Kendell's conclusion and support the finding of a melancholia syndrome. For example, Matussek et al. (27), Maes et al. (28), Parker and his coworkers $(12,29)$, Sullivan et al. (30) and Ambrosini et al. (31) identify clusters of depressed patients with distinct qualities of mood, loss of reactivity, social withdrawal, loss of energy, psychomotor abnormalities of paucity of speech and movement, vegetative signs and disturbances

Table 3. Kendell's two-depression factors

\begin{tabular}{ll}
\hline Neurotic depression & \multicolumn{1}{c}{ Psychotic depression } \\
\hline Previous anxiety symptoms & Gross disturbance in food intake \\
Previous subjective tension & Gross disturbance in weight \\
Previous 'hysterical' symptoms & Severe insomnia \\
Childhood neurotic & Retarded activity \\
traits/suicidal feelings & Abnormal quantity of speech \\
Previous obsessional symptoms & Social withdrawal \\
& Delusions (guilt, self-reproach, \\
& worthlessness) \\
& Ideas of reference, suspiciousness, \\
& persecutory delusions \\
\hline
\end{tabular}


in circadian rhythms, and suicidal thoughts. Such patients have a poor prognosis for marginally effective interventions and require intensive biologically based treatments $(1,11,27-31)$.

\section{Verification by laboratory tests}

Few laboratory tests are associated with abnormal behaviors. Aside from serological tests for syphilis and systemic examinations for infection or metabolic error, the EEG has been the most helpful test of a behavior syndrome. Single inter-ictal recordings exhibit seizure rhythms in $50-55 \%$ of patients with well-defined epilepsy. Two recordings increase sensitivity to $92 \%$ (32). It is difficult to conceive of a medical examination that considers epilepsy without examining EEG recordings. This standard is useful in discussing the role of laboratory tests in melancholia.

Hypercortisolemia is a feature of pituitary and adrenal pathology. In the search for normative standards, highly abnormal levels were found in melancholic depressed patients. The finding stimulated an interest in cortisol physiology and led to the development of the DST (22).

Elevated levels of cortisol, loss of diurnal rhythmicity, and impaired suppression of cortisol by dexamethasone were well documented in severely depressed patients, especially in those with weight loss, agitation, suicide risk, and psychosis. With successful antidepressant treatment, plasma cortisol levels fell to the levels of normal subjects. Carroll concluded that: 'The suppression test distinguished clearly between the depressed and the control patients who could not be distinguished simply on the basis of their diurnal plasma cortisol levels' (33).

By 1976, the DST was established as a measure of severe depression. Greater Hypothalmic-Pituitary-Adrenal (HPA) activity before dexamethasone and less complete HPA suppression following its administration was reported in 42 patients with 'endogenous depression' compared with 42 patients with other psychiatric disorders. Patients with two or more abnormal cortisol values after dexamethasone were correctly identified as being endogenously depressed (34).

By 1981, the DST was validated '.... as a test for the diagnosis of melancholia (endogenous depression)' in 438 patients using a cut-off level of $5 \mu \mathrm{g} / \mathrm{dl}$ plasma cortisol after $1 \mathrm{mg}$ dexamethasone administration (35). Two blood samples taken at 16.00 and $21.00 \mathrm{~h}$ after dexamethasone administered at $21.00 \mathrm{~h}$ the night before 'detected $98 \%$ of the abnormal test results. This version of the DST identified melancholic patients with a sensitivity of $67 \%$ and a specificity of $96 \%$.
Doing the test properly, however, is difficult. Each laboratory needs to establish local normative standards. Monitoring plasma dexamethasone levels is necessary as the systemic response is sensitive to dexamethasone dosage and serum levels. Pregnancy, end-stage dementia, substantial weight loss, and interference from other agents (e.g. steroids, some anticonvulsants) affect the test and are necessarily considered in assessing its results.

The DST was widely recognized as a state marker, but it was also examined for its correlation with clinical diagnoses. The DST is commonly abnormal in patients with delusional (psychotic) depression. A meta-analysis of 14 studies comparing DST results in psychotic and non-psychotic depressed patients found the non-suppression rate to be substantially higher in psychotic depressed patients (36). Patients with schizophrenia typically do not show abnormal cortisol levels, arguing that the high levels are characteristic not of psychosis, but of mood disorder (1).

The frequency of non-suppression in manic patients varied from $0 \%$ to $70 \%$ with the highest frequency in mixed bipolar disorder (1).

Depressed patients with abnormal DST have a higher risk for suicide. In a 15-year follow-up study the suicide risk in those with an abnormal DST was $27 \%$ compared with $3 \%$ among patients with a normal DST. A review of 101 patients re-examined over 2 years confirmed the higher risk for suicide and indicated hospitalization for suicide risk in those with abnormal DST. Patients with abnormal cortisol metabolism are more likely to make suicide attempts (1).

DSM-III was introduced at the time that the DST was being assessed, and its role as a diagnostic test was examined. By 1985, reviewers concluded that:

The sensitivity of the DST in major depression is limited (about $44 \%$ in over 5000 cases) but is higher in psychotic affective disorders and mixed manic-depressive states $(67 \%$ to $78 \%)$. The high specificity of the DST vs control subjects (over 90\%) is not maintained vs other psychiatric disorders (77\% specificity overall), and acute 'distress' may contribute to non-suppression of cortisol (37).

These authors concluded:

The test may have power in differentiating severe melancholic depression, mania, or acute psychosis from chronic psychosis (87\% specificity) or dysthymia $(77 \%$ specificity).

The DST was discarded from the clinic and in psychiatric research. Yet, as recently as 1996, Rush et al. (38), in a large study of patients meeting 
criteria for both unipolar and bipolar depression by RDC criteria, reported cortisol non-suppression in $35 \%$ of endogenously ill out-patients and in $9 \%$ of non-endogenously ill. Among in-patients, nonsuppression was found in $62 \%$ and $19 \%$ respectively. The sensitivity was assessed as $40 \%$, the specificity at $90 \%$. In placebo responders, $10 \%$ are DST non-suppressors and 70\% are suppressors (1).

The weight of the evidence indicates that the DST is a valid test of the presence of a severe depressive mood disorder, especially in those meeting criteria for psychotic and melancholic depression. Like the EEG in seizure disorders, measures of cortisol physiology are not simple tests, but offer a useful criterion in defining an illness. A positive test assures the diagnosis; a negative test encourages further assessment. Additional measures as the thyroid stimulating hormone (TSH) response to thyrotropin-releasing hormone (TRH) and the sleep EEG offer further validation criteria (1).

The dexamethasone-suppressed corticotropinreleasing hormone (DEX/CRH) measure of cortisol physiology improves sensitivity (1). It took more than half a century of experience from the descriptions of the human EEG by Hans Berger in the 1930s to achieve the quantitative digital computer and video-assisted EEG diagnoses of today. Improvements in measures of cortisol and neuroendocrine physiology offer a similar opportunity to design better laboratory test criteria for melancholia.

Although less well codified, studies of EEG sleep characteristics consistently find greater abnormalities in hospitalized, more severely ill patients exhibiting features of endogenous depression. Sleep studies, are difficult to apply clinically, but have heuristic research value in defining a melancholic depression (1).

\section{Verification by treatment}

The development of highly efficient antibiotics for bacterial infections, and vitamin and hormone replacements for dietary and hormone deficiencies verified a clinical diagnosis. The astonishing efficacy of ECT in remitting melancholia also supports the diagnosis. Melancholia remits with greater than $90 \%$ efficiency within 3 weeks with bilateral ECT $(39,40)$. Critics will note that ECT is as effective in delirious mania and catatonia as it is in melancholia, and will argue that these actions are too broad to verify a diagnosis. Patients with mania, however, almost always have episodes of depressive illness. Over their life-time, they experience depression more often than episodes of mania. Catatonia is commonly defined in patients with mood disor- ders, and almost all patients in a melancholic or manic episode exhibit some features of catatonia (e.g. automatic obedience, ambitendency) (3, 4). Furthermore, the broad action of penicillin in bacterial and spirochaetal infections does not invalidate its specificity for either condition.

The multi-site collaborative study of continuation ECT and continuation pharmacotherapy (known as CORE) used bilateral ECT in patients meeting rigorous criteria for unipolar major depression. It reported an overall remission rate of $87 \%$ among treatment completers, with an astonishing $95 \%$ rate for the $30 \%$ of patients identified as psychotic depressed based on Structured Clinical Interview for DSM-IV (SCID) criteria $(41,42)$. Although characterized as meeting criteria for major depression, the major part of the CORE sample meets the criteria for melancholia based on severity of the mood disorder, high incidence of psychosis and suicide risk, and failure to respond to multiple medication trials. A posthoc analysis of the items rated on the Hamilton Depression Rating Scale (HAMD) ${ }_{24}$, SCID, and Inventory of Deppressive Symptomatology (IDS) scales finds that more than $60 \%$ meet symptom criteria for melancholia.

Lesser remission rates of $50-60 \%$ with ECT in hospitalized unipolar depressed patients are still reported, but are understood to result from inefficient treatment methods (43). Successful ECT also reverses the hormone imbalances seen in depressive illness. Carroll (33) reported a normalized DST in five patients treated with ECT, with reversion to abnormality heralding relapse. The same reversal in other hormone tests has been observed and these experiences are the basis for a neuroendocrine explanation of the mode of action of induced seizures in melancholia $(39,44)$.

Broad pharmacodynamic spectrum antidepressants are more effective in melancholic than in nonmelancholic patients. Lithium moderates abnormal mood and reduces suicidal drive, and is the most effective augmenting agent in the treatment of acute depressive illness when the patient is melancholic. It is efficient as continuation therapy for melancholic patients, especially when combined with the tricyclic nortriptyline $(1,11,42)$.

Tricyclic antidepressants effectively relieve melancholic depression but do so less efficiently than ECT. The imbalance is seen in the relative inefficacy of TCA in psychotic melancholic patients where remission rates are low $(1,11)$.

Although the more recently introduced selectiveserotonin reuptake inhibitor and similar agents are widely recommended as the first agents in treatment algorithms for major depression, their overall 
$30-40 \%$ remission rates differ only minimally from placebo (45). They are much less effective in hospitalized patients, those more likely to be suffering from melancholic depression. These experiences speak against their specificity in the pathophysiology of melancholia. By contrast, the extraordinary efficacy of ECT in inducing remission, reducing suicide risk, and reversing endocrine abnormalities marks the mechanism of action of induced seizures as an essential target for studies of the pathophysiology of melancholia (44).

\section{Unipolar-bipolar distinction}

Patients with mood disorder are now dichotomized by a history of manic or hypomanic episodes into recurrent depressive or bipolar disorder. When depressive illness is grouped by criteria of melancholia, however, the unipolar/bipolar dichotomy fails. No characteristic psychopathology separates the melancholic patient with a history of mania or hypomania from one without such a history. The illnesses are commonly recurrent and in succeeding episodes $70 \%$ of patients initially classified as bipolar disorder meet criteria for major depression; and, in the course of a major depressive illness, $10 \%$ exhibit episodes of mania and over half exhibit features of mania when depressed (1).

In an extensive analysis of unipolar and bipolar depression examining the course, epidemiology, family history, physiological measures, incidence of clinical signs of anxiety, anger, psychomotor agitation, mood lability, sleep time, and pain sensitivity Goodwin and Jamison (11) were unable to identify distinguishing characteristics. They found the breadth of differences and heterogeneity impressive, and yet they concluded:

Taken together, the data suggest that they (mania, depression) are best considered as two subgroups of manic-depressive illness rather than separate and distinct illnesses. The available data also support a continuum model, with 'pure' bipolar illness at one end and unipolar illness at the other.

Others also fail to find psychopathological separation points for the two conditions (46-48). Historically, the two conditions were identified as facets of a condition of circular insanity by French psychopathologists Falret (24) and Baillarger (49). Their descriptions were adopted by Kraepelin (50) in the concept of manic-depressive psychosis that has been a mainstay of classification until DSM-III. Demarcating melancholia as a defined depressive mood disorder encourages the earlier views of a single psychopathology for mania and depression.
In conclusion, the present classification of mood disorders is arbitrary, divided into a multitude of presumed disorders with imprecise criteria. The formulations do not encourage effective therapy or definitive research. Patients meeting criteria for single major depression are said to respond to the same treatment algorithm, with the more severe needing higher doses or drug combinations, but not different approaches. The putative unipolar and bipolar disorders are said to require different algorithms.

The evidence is the opposite: melancholia can be distinguished from other depressive mood disorders by its characteristic psychopathology. The unipolar/ bipolar dichotomy is not supported when the criteria for melancholia are used to classify depressive illness. The treatment algorithm for melancholia differs from that of non-melancholic depressions, but the treatment algorithms for melancholia with or without mania are similar. We find compelling evidence that hypercortisolemia is characteristic of severely ill depressed patients, and tests of this hormone (and other hormones) should be considered in the classification and the management of patients with depressive mood disorders. Various treatments - lithium, tricyclic antidepressants, and ECT - effectively relieve both the depressive and manic phases of the syndrome. The expert treatment algorithms thus require substantial revision if they are to claim to be 'evidence based'.

We propose the demarcation of a syndrome of melancholia as the best opportunity to develop homogeneous study populations, more effective treatment algorithms, better understanding of the pathophysiology of mood disorders, and reducing the social burden of 'therapy-resistant' depressed patients.

\section{References}

1. TAYlor MA, FinK M. Melancholia: the diagnosis, pathophysiology and treatment of depressive illness. Cambridge, UK: Cambridge University Press, 2006.

2. Rush AJ, TRIVEd MH, WisNIEWSKI SR et al. Bupropion-SR, sertraline, or venlafaxine-XR after failure of SSRIs for depression. N Engl J Med 2006;354:1231-1242.

3. Robins E, Guze SB. Establishment of diagnostic validity in psychiatric illness: its application to schizophrenia. Am J Psychiatry 1970;126:983-987.

4. FinK M, TAYlor MA. Catatonia: a clinician's guide to diagnosis and treatment. Cambridge, UK. Cambridge University Press, 2003.

5. TAYLOR MA, Fink M. Catatonia in psychiatric classification: a home of its own. Am J Psychiatry 2003;160:1233-1241.

6. Stewart J, Klein DF, McGrath P, Quitkin F. Heterogeneity: the hobgoblin of psychiatric diagnosis; dissecting atypical depression: a roadmap to homogeneity? Acta Psychiatr Scand Suppl 2007;433:58-71. 
7. Hopewell-Ash EL. Melancholia in everyday practice. London: John Bale Sons \& Danielsson, 1934.

8. Lewis AJ. Melancholia: a historical review. J Ment Sci 1934;80:1-42.

9. Schmidt-Degenhard M. Melancholie und depression. Stuttgart: Verlag W. Kohlhammer, 1983.

10. JACKSON SW. Melancholia and depression from Hippocrates to modern times. New Haven, CT: Yale University Press, 1986.

11. Goodwin FK, Jamison KR. Manic-depressive illness. New York: Oxford University Press, 1990; 227-244.

12. Parker G, Hadzi-Pavlovic D. Melancholia: a disorder of movement and mood. Cambridge, UK: Cambridge University Press, 1996.

13. KLEIN DF, FINK M. Psychiatric reaction patterns to imipramine. Am J Psychiatry 1962;119:432-438.

14. KLeIN DF, Fink M. Behavioral reaction patterns with phenothiazines. Arch Gen Psychiatry 1962;7:449-459.

15. Fink M, Klein DF, Kramer J. Clinical efficacy of chlorpromazine-procyclidine combination, imipramine and placebo in depressive disorders. Psychopharmacologia 1965;7:27-36.

16. KLEIN DF. Delineation of two-drug-responsive anxiety syndromes. Psychopharmacologia 1964;5:397-408.

17. Nelson JC, Charney DS. The symptoms of major depressive illness. Am J Psychiatry 1981;138:1-13.

18. Sandifer MG, Hordern A, Timbury GC, Green LM. Similarities and differences in patient evaluation by U.S. and U.K. psychiatrists. Am J Psychiatry 1969;126:206-212.

19. Settzer RL, Fleiss JL. A re-analysis of the reliability of psychiatric diagnosis. Brit J Psychiatry 1974;125:341-347.

20. Shorter E. A history of psychiatry from the era of the asylum to the age of Prozac. New York: John Wiley \& Sons, 1997.

21. World Health Organization. The ICD-10 classification of mental and behavioral disorders. Geneva, Switzerland: WHO, 1992.

22. Davies BJ, Carroll BJ, Mowbray RM. Depressive illness: some research studies. Springfield, IL: C.C. Thomas, 1972.

23. FINK M. Should the dexamethasone suppression test be resurrected? Acta Psychiatr Scand 2005;112:245-249.

24. Falret JP. Mémoire sur la folie circulaire. Bulletin de l'Academic nationale de Médicine 1854;19:382-415.

25. Kendell RE. The classification of depressive illness. London: Oxford University Press, 1968.

26. Kendell RE. The classification of depressions: a review of contemporary confusion. Br J Psychiatry 1976;129: $15-28$.

27. Matussek P, Soldner M, Nagel D. Identification of the endogenous depressive syndrome based on the symptoms and the characteristics of the course. $\mathrm{Br} \mathrm{J}$ Psychiatry 1981;138:361-372.

28. Maes M, Cosyns P, Maes L, D'Hondt P, Schotte C. Clinical subtypes of unipolar depression, Part I: a validation of the vital and nonvital clusters. Psychiatry Res 1990;34:29-41.

29. Parker G, Hadzi-Pavlovic D, Wilhelm K et al. Defining melancholia: properties of a refined sign-based measure. $\mathrm{Br}$ J Psychiatry 1994;164:316-326.

30. Sullivan PF, Prescott CA, Kendler KS. The subtypes of major depression in a twin registry. $\mathbf{J}$ Affect Disord 2002;68:273-284
31. Ambrosini PJ, Bennett DS, Cleland CM, Haslam N. Taxonicity of adolescent melancholia: a categorical or dimensional construct? J Psychiatr Res 2002;36:247-256.

32. Parra J, Augustijn PB, Geeerts Y, Van Emde Boas W. Classification of epileptic seizures: a comparison of two systems. Epilepsia 2001;42:476-482.

33. Carroll B. Control of plasma cortisol levels in depression: studies with the dexamethasone suppression test. In: DAviES B, Carroll BJ, Mowbray RM, eds. Depressive illness: some research studies, Vol. 5. Springfield, IL: C.C. Thomas, 1972:87-149.

34. Carroll BJ, Curtis GC, Mendels J. Neuroendocrine regulation in depression, II: discrimination of depressed from nondepressed patients. Arch Gen Psychiatry 1976;33:10511058.

35. Carroll BJ, Feinberg M, Greden JF et al. A specific laboratory test for the diagnosis of melancholia. Arch Gen Psychiatry 1981;38:15-22.

36. Nelson JC, Davis JM. DST studies in psychotic depression: a meta-analysis. Am J Psychiatry 1997;154:1497-1503.

37. Arana GW, Baldessarini RJ, Ornsteen M. The dexamethasone suppression test for diagnosis and prognosis in psychiatry. Arch Gen Psychiatry 1985;42:1193-1204.

38. Rush AJ, Giles DE, Schlesser MA et al. The dexamethasone suppression test in patients with mood disorders. J Clin Psychiatry 1996;57:470-484.

39. FINK M. Convulsive therapy: theory and practice. New York: Raven Press, 1979.

40. AвRAms R. Electroconvulsive therapy, 4th edn. New York: Oxford University Press, 2002.

41. Petrides G, Fink M, Husain MM et al. ECT remission rates in psychotic versus non-psychotic depressed patients: a report from CORE. Journal of ECT 2001;17:244-253.

42. Kellner CH, Knapp RG, Petrides G et al. Continuation ECT versus pharmacotherapy for relapse prevention in major depression: a multi-site study from CORE. Arch Gen Psychiatry 2006;63:1337-44.

43. Sackeim HA, Prudic J, Devanand DP et al. A prospective, randomized, double-blind comparison of bilateral and right unilateral electroconvulsive therapy at different stimulus intensities. Arch Gen Psychiatry 2000;57:425-434.

44. FINK M. Electroshock revisited. Am Sci 2000;88:162-167.

45. Khan A, Kolts RL, Rapaport MH, Krishnan KR, Brodhead AE, BRown WA. Magnitude of placebo response and drugplacebo differences across psychiatric disorders. Psychol Med 2005;35:743-749.

46. Akiskal HS, Benazzi F. Validating Kraepelin's two types of depressive mixed states: 'depression with flight of ideas' and 'excited depression'. World J Biol Psychiatry 2004;5:107-113.

47. BenAzZI F. Melancholic outpatient depression in bipolar-II vs. unipolar. Prog Neuro-psychopharm Biol Psychiatry 2004;28:481-485.

48. Benazzi F. Intra-episode hypomanic symptoms during major depression and their correlates. Psychiatry Clin Neurosci 2004;58:289-294.

49. Baillarger J. De la folie à double forme. Ann Med Psychol 1854;6:367-391.

50. KRAEPelin E. Manic-depressive insanity and paranoia. Barclay RM, translator, Robertson GM, ed. Edinburgh: E\&S Livingstone, 1921; repr. New York: Arno Press, 1976. 